\title{
Short - term load forecasting of power system
}

\author{
Zongheng Jiang \\ North China Electric Power University (Baoding), Baoding 071000, China \\ 271856330@qq.com
}

Keywords: the partial least squares regression analysis, ARMA time series, BP neural network.

\begin{abstract}
Short-term load forecasting is of great significance to the operation and analysis of power system to improve the accuracy of load forecasting. An Important Means to Guarantee the Scientific Decision of Power System Optimization. This paper aims to analyze the load fluctuation of the two regions and the relationship between meteorological factors, holiday factors and periodicity, and set up the least squares regression analysis model, time series model and BP neural network model to short - term load forecasting of power system.
\end{abstract}

\section{Introduction}

First, select the maximum daily temperature, the daily minimum temperature, the daily average temperature, the daily relative humidity, the daily precipitation as the independent variable, the daily maximum load, the daily minimum load, the daily average load as the dependent variable, the establishment of partial least squares regression model. Then we draw the change curve of electricity consumption in 2014. According to the trend of curve change, ARMA time series model is used to model the electricity load. Taking into account the frequent weather changes in the region, heavy rain, cooling or sustained high temperature weather will have a great impact on load forecasting. Therefore, BP neural network is used to input 10 influencing factors of electricity load and meteorological factors in the corresponding 5 weeks, and the corresponding prediction results are modeled as output. According to the results, the model we have established is in good agreement with the actual situation.

\section{Assumptions}

1. Assuming that the temperature difference between day and night is closer, the temperature data given can reflect the day Where the temperature changes.

2. Assuming that the meteorological factors given are the main factors, ignoring the price of electricity, the living standards and other factors on the use of the effect of electrical load.

3. Assume that all data are raw data and that the source is reliable.

4. Assuming that the data in the given time period are the statistical results under normal circumstances, that is, due to accidental factors Caused by the phenomenon of power failure.

\section{Forecast model of power load}

We collected data from two regions for two years, including temperature data and meteorological data. And the data were horizontal and vertical processing, to ensure the correctness of the data. Then, the ARMA time series model, partial least squares regression model and BP neural network model are established respectively considering different influencing factors. Finally, the prediction results are verified.

\subsection{Establishment of Partial Least Squares Regression Analysis Model}

In this paper, the influence of each meteorological factor on the power load is taken into account. The maximum temperature, daily minimum temperature, daily average temperature, daily relative humidity and daily precipitation are taken as the independent variables in the area 1 , The daily minimum load, daily average load as the dependent variable, the partial least squares regression analysis model[1] was established, and the relationship between daily maximum load, daily minimum load, daily average load and meteorological factors was analyzed. 
According to the formula, into the data to calculate, the result is:

$$
\left\{\begin{array}{l}
y_{1}=\left(5.5776+0.0723 x_{1}+0.0635 x_{2}+0.0805 x_{3}-0.0156_{4}+0.0101 x_{5}\right) \times 10^{3} \\
y_{2}=\left(2.3896+0.0534 x_{1}+0.0469 x_{2}+0.0596 x_{3}-0.0136 x_{4}+0.0081 x_{5}\right) \times 10^{3} \\
y_{3}=\left(4.2048+0.0652 x_{1}+0.0573 x_{2}+0.0728 x_{3}-0.0159 x_{4}+0.0096 x_{5}\right) \times 10^{3}
\end{array}\right.
$$

Where $\mathrm{x} 1$ is the maximum daily temperature, $\mathrm{x} 2$ is the lowest temperature, $\mathrm{x} 3$ is the daily average temperature, $\mathrm{x} 4$ is the daily relative humidity, $\mathrm{x} 5$ is the daily precipitation. $\mathrm{Y} 1$ is the highest daily load, y2 is the daily minimum load, y3 is the daily average load.

It can be seen that the maximum temperature, the minimum temperature and the average temperature have a significant effect on the electricity load, while the relative humidity and precipitation have a weaker effect on the electricity load.

\subsection{ARMA time series Model}

Through the analysis of electricity load, we find that there is obvious periodic law of this time series. In response to this phenomenon, we selected the corresponding seasonal model to study the trend of changes in power load.

Using the time series[2] method to predict the load of the three weeks before January 11, 2014, the load of January 17, 2014 to January 17, 2014 is predicted. By calculating the autocorrelation function and the partial correlation function, $d=1$. Using the order of AIC criterion, $q=1, p=6$, the ARIMA $(1,1,6)$ model is obtained, and the maximum likelihood estimation is obtained

$$
\begin{gathered}
\theta=(-0.8648)^{T} \\
\varphi=(1.6294,1.2461,0.9459,0.6885,0.4722,0.2177)^{T}
\end{gathered}
$$

The model is:

$$
\begin{aligned}
& (1+0.8648 B)(1-B) X_{t} \\
& =\left(1-1.6294 B-1.2461 B^{2}-0.9459 B^{3}-0.6885 B^{4}-0.4722 B^{5}-0.2177 B^{6}\right) \varepsilon_{t}
\end{aligned}
$$

After $\chi_{2}$ testing, accept $\mathrm{H} 0$, that $\varepsilon_{t}$ is white noise, the model passed the test. The predicted curve is compared with the actual curve as shown in Figure 1.

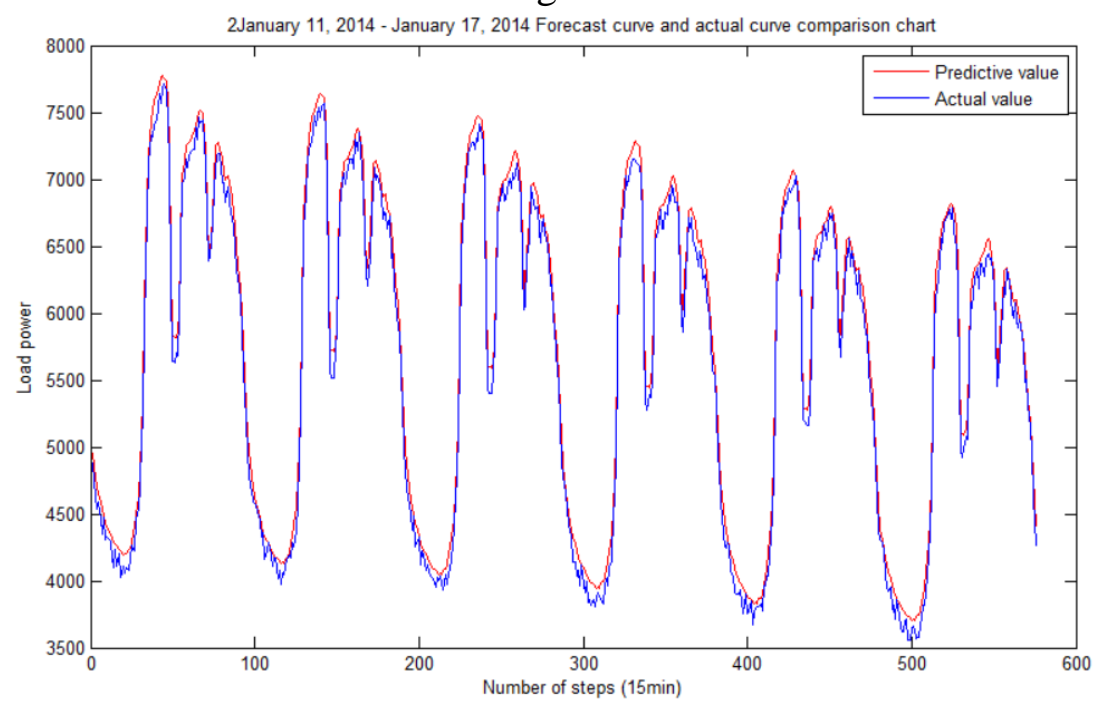

Figure 1. Predicted curve compared with the actual curve

\subsection{BP neural network}

In the short-term load forecasting of the power system, the meteorological factors are one of the important factors that affect the load change and the prediction accuracy. Especially for areas with frequent weather changes, heavy rain, cooling or continuous high temperature weather will have a great impact on load forecasting. In order to consider the influence of meteorological factors while 
considering the influence of meteorological factors, we use BP neural network model to solve the load forecasting problem of meteorological factors in order to improve the prediction accuracy under weather conditions.

BP neural network [3] is generally composed of three layers:

1. Output layer: 10 weeks before the corresponding period of electricity load and meteorological factors a total of 10 influencing factors for input.

2. Implicit layer: The number of neurons in the hidden layer is set to 8 .

3. Output layer: the corresponding forecast results as output, the number of output layer 1.

Then we use BP neural network to predict the data, the results are as shown in Figure 2.

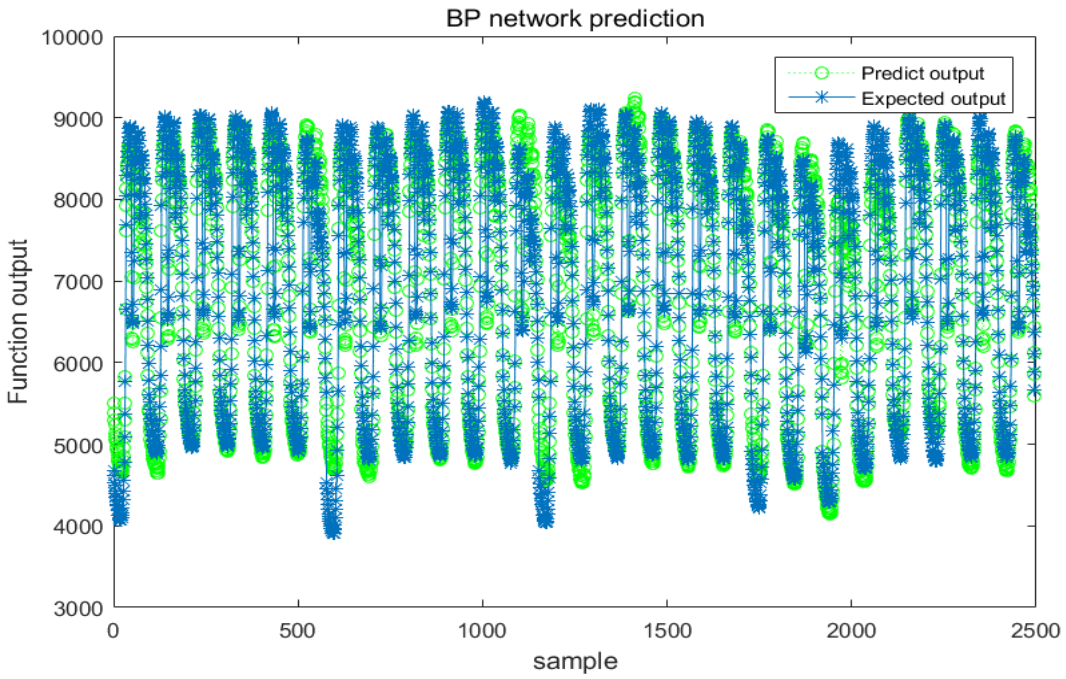

Figure 2. BP neural network to predict the data

\section{The advantages and disadvantages of the model}

\subsection{Advantages of the model}

1. In the analysis of the data, found that some data missing and abnormal, the data were reasonable Effective addition and modification, to achieve the purpose of eliminating bad data.

2. In the process of forecasting the model, we do not use the general data of each time period as a class, and is the use of time series for decomposition, which has been a perfect model.

\subsection{The shortcomings of the model}

Not taking into account factors such as the living standards of the residents, the level of regional development and other factors.

\section{Conclusion}

The model established in this paper has universal applicability, not only can be used for large data processing, but also can be used In the short-term load forecasting of the power system, it can also be used in the periodic forecasting problem such as water conservancy.

\section{References}

[1]Geladi P, Kowalski B R. Partial least-squares regression: a tutorial [J]. Analytica chimica acta, 1986, 185: 1-17.

[2]McLeod A I, Li W K. Diagnostic checking ARMA time series models using squared - residual autocorrelations [J]. Journal of Time Series Analysis, 1983, 4(4): 269-273.

[3]Jin W, Li Z J, Wei L S, et al. The improvements of BP neural network learning algorithm[C] //Signal Processing Proceedings, 2000. WCCC-ICSP 2000. 5th International Conference on. IEEE, 2000, 3: 1647-1649. 